\title{
A case-control study of the association between psychosocial factors and the occurrence of laryngeal cancer
}

\author{
CHAO WANG ${ }^{1}$, GUOLIANG FAN ${ }^{2}$, RUI XU ${ }^{1}$, JINGTING WANG ${ }^{1}$, LINA WANG $^{3}$, LU ZHANG $^{1}$ and QIUYING LI ${ }^{1}$ \\ ${ }^{1}$ Department of Otolaryngology-Head and Neck Surgery, The Second Affiliated Hospital of Harbin Medical University, \\ Harbin, Heilongjiang 150081; ${ }^{2}$ Department of Otolaryngology, The First Hospital of Harbin, Harbin, Heilongjiang 150010; \\ ${ }^{3}$ Department of Pathophysiology, Harbin Medical University, Harbin, Heilongjiang 150081, P.R. China
}

Received February 15, 2017; Accepted July 22, 2017

DOI: $10.3892 / \mathrm{mco} .2017 .1348$

\begin{abstract}
The aim of the present study was to investigate the association between social psychological factors and the occurrence of laryngeal cancer. A 1:1 matched case-control study was conducted. The participants completed questionnaires that included general information, such as the Life Event Scale, the Social Support Rating Scale, and the Eysenck Personality Questionnaire (EPQ). Scores were compared between the groups using paired t-tests and Wilcoxon's signed-rank tests. No significant difference in the psychoticism scale of the EPQ was observed between the two groups $(\mathrm{P}>0.05)$. However, significant differences were observed in scores on the life events and social support scales and in the remaining dimensions of the EPQ (all P-values <0.05). Positive life events may be protective factors for laryngeal cancer, whereas reduced utilization of social support may be a risk factor for laryngeal cancer.
\end{abstract}

\section{Introduction}

Laryngeal cancer is a common malignant tumor of the head and neck region, exhibiting an increasing incidence in recent years. The latest data indicated the global standardized incidence rate of laryngeal cancer to be 2.2/100,000 population (1). In China, specifically, the incidence rates in certain provinces have been reported to be $\sim 2.31 / 10$ million (2). However, this rate has increased in recent years. In modern medicine, it is important to consider not only factors such as physical chemical stimulation and pathogenic infection, but also socioeconomic factors, life and dietary habits, personality and psychological behavior (3). The majority of previous studies on laryngeal cancer have

Correspondence to: Dr Qiuying Li, Department of Otolaryngology-Head and Neck Surgery, The Second Affiliated Hospital of Harbin Medical University, 246 Xuefu Road, Harbin, Heilongjiang 150081, P.R. China

E-mail: qiuying0912@163.com

Key words: laryngeal cancer, psychosocial factors, matched case-control studies indicated that its incidence is associated with smoking, alcohol consumption, lifestyle factors and living environment $(4,5)$. However, a growing number of studies have demonstrated that stress, low social support, community alienation, chronic social isolation, and other related psychological variables, may lead to cancer development (6-8). As medicine has shifted from its reliance on the traditional biomedical model to a more multidisciplinary biological-psychological-social model, the profound impact that social psychology has on cancer risk, development and outcome, has become more apparent (5). Psychosocial factors refer to life events, coping mechanisms, social support and personal traits, among others. Through retrospective and prospective research, national and international studies have discovered that long-term and chronic stress and stressful life events may increase the risk of various tumors, such as breast $(7,9)$, prostate (10) and colorectal cancer (11). A 1:1 matched case-control study was conducted, involving 237 laryngeal cancer patients and healthy controls, in order to expand on these findings and provide evidence for the improvement of laryngeal cancer prevention and treatment through elucidating the association between certain psychosocial factors and laryngeal cancer.

\section{Subjects and methods}

Selection of subjects. Study subjects were selected among patients who had visited the Second Affiliated Hospital of Harbin Medical University (Harbin, China) between January 2010 and December 2014. Only patients with laryngeal cancer microscopically confirmed by a pathologist at the Second Affiliated Hospital of Harbin Medical University were included in the patient group, while healthy individuals who visited the hospital for routine physical examination during the same period were recruited into the control group. The individuals in the two groups were 1:1 paired. The pairing criteria were as follows: i) An age difference of $<2$ years; ii) gender and ethnic compositions were the same; and iii) the urban/suburban areas where the member of each pair lived had the same or similar transportation conditions, economic levels, living customs (e.g., diet, drinking/smoking history), cultural background, and geographical environment. The exclusion criteria for all study subjects were as follows: i) An individual or family history of mental disorders and ii) another neoplastic disease. 
Ultimately, 237 pairs were considered eligible for participation, including 194 male and 43 female pairs (sex ratio, 4.5:1; mean age, 54.2 years; age range, $40-73$ years).

Survey method and questionnaire contents. The authors assert that all procedures contributing to this study comply with the ethical standards of The Second Affiliated Hospital of Harbin Medical University (approval no. 2015-RESEARCH-206, 28 October 2015) and with the Helsinki Declaration of 1975 , as revised in 2008.

After obtaining written informed consent, each participant was interviewed face-to-face to complete the questionnaire. To reduce the statistical error introduced by having multiple investigators, all questionnaires were completed by the same investigator. The questionnaires were reviewed by specialized personnel, and the data were then entered into a computer by two researchers.

The questionnaire contents were as follows: First, the participants completed items on general information (e.g., education level, occupation, family income and household expenditure). Second, they completed the Life Event Scale (LES), which was developed by Desen Yang and Yalin Zhang (12). This scale comprises 48 commonly observed life events in China, relating to three aspects of life, namely family life (28 items), work (13 items), and social and other aspects (7 items). Furthermore, there are two blank entries for respondents to fill in events that they have experienced, but are not listed in the form. Participants were asked to list all the life events that could be recollected from birth until the malignancy diagnosis (for the patient group) or until the survey period (for the control group). Each participant was asked to evaluate each event as either good (i.e., a positive life event) or bad (i.e., a negative life event) according to his/her actual experiences, rather than according to common sense or ethics. The stress derived from those life events was then quantified using weighted composite scores according to their frequency, duration, and impact (the amount of stimulation for an event=event impact score xduration of the event points $x$ number of incidents), which is similar to the Social Readjustment Rating Scale, a measure designed by Holmes and Rahes in 1967 (13) to determine the association between life events and disease. Higher total LES scores indicate greater mental stress. For example, the higher the negative event score, the more severe the adverse effect on the respondent's physical and mental health.

Third, the participants completed the 10 items of the Social Support Rating Scale developed by Shuiyuan Xiao. This scale is used to measure the amount of social support that respondents have obtained from society. From these 10 items, total, objective support, subjective support, and support utilization scores are calculated.

Finally, participants completed the Eysenck Personality Questionnaire (EPQ) (14), which comprises scales for the following 4 dimensions of personality: extraversion (E), wherein higher scores represent greater extroversion; neuroticism (or nervosity, $\mathrm{N}$ ), wherein higher scores reflect less emotional stability; psychoticism (P), wherein higher scores indicate higher psychoticism; and lie (L), wherein higher scores are associated with a greater tendency to lie. More specifically, an extraversion score of $>15$ indicates an outgoing personality (social, longing for excitement and adventure and impulsive). By contrast, a score of $<8$ indicates an introverted personality (quiet, introspective, preferring an orderly lifestyle and emotionally stable). For the neuroticism scale, scores of $>14$ indicate an anxious, worried, and often moody personality, along with a tendency to exhibit strong emotional reactions or irrational behavior. By contrast, neuroticism scale scores of $<9$ indicate an emotionally stable personality. A high psychoticism scale score indicates loneliness, difficulty adapting to the external environment, and a tendency to engage in strange activities, regardless of the danger. Finally, for the lie scale, a score of $>18$ indicates a tendency to dissemble, which may invalidate the survey results. Therefore, any respondents with lie scale scores meeting this cut-off were excluded prior to sample pairing.

Statistical analysis. Data processing was conducted using statistical software R3.2.2 (http://cran.r-project.org/mirrors. html). Data following a normal distribution were described using means and standard deviations, while data that did not follow a normal distribution were described using medians and the lower and upper quartiles. Paired data that followed a normal distribution with homogeneity of variance were tested using paired t-tests, while Wilcoxon's signed-ranked tests were used to analyze paired data that did not follow a normal distribution. A P $<0.05$ was considered to indicate statistically significant differences.

\section{Results}

Comparison of life events between groups. The total and negative life event scores followed a normal distribution, and were thus compared by group, using t-tests (Table I). These scores were significantly higher in the patient group compared with the control group. Regarding positive life events, the data did not follow a normal distribution, and thus were tested using Wilcoxon's signed-rank tests (Table II). Notably, the patient group obtained significantly lower positive life events scores compared with subjects in the control group.

Comparison of social support between groups. According to the comparison of the Social Support Rating Scale scores between groups, it was observed that scores on the subjective support, objective support, support utilization, and total social support scales were significantly lower in the patient group compared with those in the control group (Table III).

Comparison of personality traits between groups. The extraversion and neuroticism scale scores of the patient group were significantly different from those of the control group. By contrast, the psychoticism scale score was similar between the two groups (Table IV).

\section{Discussion}

The question of whether psychological factors affect cancer development and progression has long been debated upon; this is not only of interest to scientists, but also of importance to patients. Although researchers have progressed considerably towards answering this question, no definitive conclusion has 
Table I. Comparison of negative and total life events between groups.

\begin{tabular}{lccc}
\hline Variables & Patient group & Control group & P-value \\
\hline $\begin{array}{l}\text { Negative life } \\
\text { events }\end{array}$ & $79.32 \pm 13.503$ & $54.07 \pm 18.516$ & $<0.001$ \\
$\begin{array}{l}\text { Total life } \\
\text { events }\end{array}$ & $147.2 \pm 20.397$ & $134.1 \pm 16.616$ & $<0.001$ \\
\hline
\end{tabular}

Values are presented as mean \pm standard deviation.

yet been reached. Garssen, upon summarizing 30 years of research, identified stressful life events, social relationships, distress levels and the number of psychological problems, having a psychiatric diagnosis, repression, coping style, and personality factors, loss events, and locus of control, as potential factors (15). Some studies have further demonstrated that psychosocial factors are associated with the occurrence of cancer $(16,17)$, while other studies considered them to be associated with cancer prognosis $(18,19)$, and even recurrence and metastasis $(20,21)$. To further advance our understanding of this association, we investigated the impact of life events and social support on the occurrence of laryngeal cancer and observed that the two groups exhibited significant differences in terms of scores on the life events and social support scales, and on all dimensions of the EPQ $(\mathrm{P}<0.05)$, except the psychoticism scale.

In the present study, the total and negative life event scores of the patient groups were found to be remarkably and significantly higher compared with those of the control group. Goodkin et al reported similar findings, in that the number of stressful life events was associated with cancer progression (22), albeit weakly. Another study investigating the impact of bereavement on cancer mortality classified cancer according to four types, namely lung, stomach, breast, and 'other'. No effect of bereavement on cancer mortality was observed among women, while in men, bereavement predicted a higher cancer mortality rate. The higher mortality rate in bereaved men was observed for only those with lung and 'other' cancers. The bereavement effect on cancer was highest for the younger group of men (aged 35-64 years), and entirely absent for the oldest group (age 75-84 years) (23). Overall, the results for life events suggest that, compared with the healthy population, patients with laryngeal cancer experienced greater mental and psychological stress and more negative emotions prior to their illness. This allows us to tentatively infer that considerable psychological stress and negative emotions may be a risk factor for laryngeal cancer. Several studies have indicated that negative life events (such as poor marital quality, loneliness, depressed mood, dissatisfaction with social support, unemployment and bereavement) affect immune system functioning; specifically, they cause immune suppression. However, the association among stress, the immune system and disease has not been fully elucidated. Negative life events may be associated with a reduction of natural killer (NK) cytotoxicity.

Regarding social support, we found that patients with laryngeal cancer obtained significantly lower scores on objective support, subjective support, and support utilization scales compared with the control group. Shaffer et al similarly found that male medical students who developed cancer while being followed up over 16-32 years had lower scores on closeness to their fathers at initial assessment, as compared to the entire group or the healthy subgroup (24). Another study reported that women with few relationships, particularly friends, and those experiencing feelings of social isolation, were at higher risks of developing cancer and dying from cancer; notably, this was not found in men (25). Levy et al found that better perceived social support was associated with a longer disease-free interval within a subgroup of women who exhibited recurrence (26). An impressive study in this area was conducted by a Canadian study group, Maunsell et al, who found a type of dose-response relationship for social support and cancer survival rate; survival rates at 7 years increased stepwise in women reporting none, one, or at least two types of confidants (27). Our findings suggest that laryngeal cancer patients lacked social assistance and support, both subjectively and objectively, compared with healthy individuals. As a result, laryngeal cancer patients were more prone to psychological and mental isolation and helplessness, which suggests that reduced social support may be a predisposing factor for laryngeal cancer. Social support is considered to promote biological or behavioral adaptations under conditions of stress. This, in turn, may lead to positive effects on immune functioning and may limit the effects of stress-related endocrine changes that are possibly associated with tumor proliferation. This may result in better treatment compliance and the adoption of better health behaviors, which will generally exert positive effects on overall physical condition.

Regarding the EPQ dimensions, the extraversion scale scores of the patient and control groups were close to 15 and 8, respectively, and these differences were significant. This result confirms that, compared with healthy individuals, patients with laryngeal cancer appear to prefer excitement and are more prone to emotional impulsiveness. Similarly, the neuroticism scale scores of the patient and control groups were close to 14 and 9, respectively, which was also a significant difference. This suggests that, compared with healthy individuals, patients with laryngeal cancer are more prone to anxiety and emotional instability. Somewhat relatedly, one population-based prospective cohort study found a significant association between neuroticism and risk of death, particularly among women (28). Furthermore, a cross-sectional analysis from the Miyagi Cohort Study demonstrated that the personality traits of extraversion, neuroticism and psychoticism were significantly associated with attendance of gastric cancer screenings (29). van den Bergh et al reported that higher neurotic personality scores were associated with unfavorable effects for patients with early prostate cancer (30), while another study among cancer patients, non-cancer patients, and healthy individuals in a Japanese sample, indicated that the health-related quality of life score of individuals with tolerable/tolerant type personalities (high $\mathrm{E}$, low $\mathrm{N}$ and high $\mathrm{P}$ scores) was higher compared with that of individuals with intolerable/intolerant type personalities (low E, high $\mathrm{N}$ and low $\mathrm{P}$ scores) and unclassified type personalities (31); notably, no differences in psychoticism scale scores were observed between the two groups. It was previously hypothesized that psychological stress may reduce 
Table II. Comparison of positive life events between groups.

\begin{tabular}{lccccr}
\hline Variables & Minimum & $\mathrm{P}_{25}$ & Median & $\mathrm{P}_{75}$ & Maximum \\
\hline Patient group & 45 & 58 & 67 & 79 & 89 \\
Control group & 45 & 79 & 89 & 90 & 103 \\
\hline
\end{tabular}

Table III. Comparison of social support between groups.

\begin{tabular}{|c|c|c|c|c|c|c|}
\hline Variables & Minimum & $\mathrm{P}_{25}$ & Median & $\mathrm{P}_{75}$ & Maximum & P-value \\
\hline Subjective social support & & & & & & $<0.001$ \\
\hline Patient group & 7 & 10 & 11 & 13 & 26 & \\
\hline Control group & 10 & 12 & 15 & 18 & 28 & \\
\hline Objective social support & & & & & & $<0.001$ \\
\hline Patient group & 3 & 5 & 6 & 7 & 12 & \\
\hline Control group & 4 & 7 & 8 & 9 & 15 & \\
\hline Social support utilization & & & & & & $<0.001$ \\
\hline Patient group & 3 & 5 & 6 & 6 & 8 & \\
\hline Control group & 3 & 6 & 6 & 7 & 9 & \\
\hline Total social support & & & & & & $<0.001$ \\
\hline Patient group & 13 & 20 & 23 & 27 & 46 & \\
\hline Control group & 13 & 24 & 28 & 33 & 52 & \\
\hline
\end{tabular}

Table IV. Comparison of personality traits between groups.

\begin{tabular}{|c|c|c|c|c|c|c|}
\hline Variables & Minimum & $\mathrm{P}_{25}$ & Median & $\mathrm{P}_{75}$ & Maximum & P-value \\
\hline Extraversion & & & & & & $<0.001$ \\
\hline Patient group & 6 & 8 & 9 & 13 & 19 & \\
\hline Control group & 6 & 11 & 13 & 14 & 25 & \\
\hline Neuroticism & & & & & & $<0.001$ \\
\hline Patient group & 3 & 9 & 10 & 13 & 23 & \\
\hline Control group & 3 & 6 & 7 & 9 & 18 & \\
\hline Psychoticism & & & & & & 0.321 \\
\hline Patient group & 3 & 6 & 8 & 10 & 16 & \\
\hline Control group & 2 & 6 & 8 & 9 & 12 & \\
\hline
\end{tabular}

NK cell activity (32). Psychological stress is also associated with the following two important processes for carcinogenesis: Poorer repair of damaged DNA and alterations in apoptosis, which play a role in malignant diseases. Conversely, the possibility that psychological interventions may enhance immune functioning and survival among cancer patients clearly merits further exploration, as does the evidence suggesting that social support may be a key psychological mediator.

The present study demonstrated that significant life and mental stress, proneness to anxiety and emotional instability are risk factors for laryngeal cancer, whereas sufficient social support and high utilization of social support are protective factors. Palermo et al systematically analyzed the effect of psychosocial factors on various stages of tumor development; they believed that psychosocial factors may increase suscepti- bility to cancer by altering the state of the body (e.g., increased possibility of genetic mutation, decreased DNA repairing capability and reduced immune function) (33). Hofs $\varnothing$ et al (34) noted that mental status was closely associated with the functioning of the nervous, endocrine and immune systems. Additionally, mental disorders, such as depression, may alter the stress levels and immune function of the body through the neuroendocrine immunomodulation system, thereby affecting tumor occurrence and progression $(34,35)$. In summary, several psycho-physiological mechanisms were found to be relevant. First, participants with high emotional constraint and/or high negative affectivity respond to stress with greater increases in heart rate, blood pressure and sweating, compared with low-anxiety, non-constrained individuals, and they also have higher basal cortisol levels. Second, decreased monocyte 
counts, elevated serum eosinophils and hyperglycemia have been found in low-anxiety/high-constraint individuals, when compared to their low-anxiety/low-constraint counterparts. This suggests that the elevation of another stress hormone, adrenocorticotropic hormone, the stimulant to cortisol secretion, and $\beta$-endorphin are both derived from a large precursor molecule, pro-opiomelanocortin, and both are released within min of exposure to mental stress. Therefore, mental stress is associated with physiological processes characteristic of emotionally constrained patients. Third, stress-induced immunosuppression is more pronounced with high emotional constraint and control over one's feelings rather than with low emotional constraint and lack of control. These physiological stress responses are known to exert deleterious health effects; however, the pathways through which they may adversely affect cancer progression remain under investigation. Furthermore, emotional distress and the psychological processes regulating it may also affect cancer progression indirectly through affecting health behavior.

The present study has several limitations. First, the interactions between the investigated factors were not considered. Second, due to different geographic populations having different dispositions, this study surveyed populations only from northern China. This may have resulted in some degree of bias in the results. Due to the abovementioned reasons and certain factors that have not been taken into consideration, it is difficult to establish with certainty whether the psychosocial factors in this study per se are risk factors for laryngeal cancer based on our study findings alone.

However, our results suggest that harmonic social relations and less dramatic mood swings may help reduce the risk of laryngeal cancer; otherwise, this risk increases. Therefore, positive social support should be provided to groups exhibiting depressed mood, in order to prevent the development of serious physical illnesses. Moreover, warning should be given to high-risk groups, including those with higher proneness to anxiety and emotional instability, to help in the prevention of adverse consequences. Such warnings could be paired with behavioral interventions to change insalubrious lifestyles and unhealthy living habits, which may make a positive contribution to the prevention and treatment of laryngeal cancer.

\section{Acknowledgements}

The authors would like to thank all the participants and their families for compiling the questionnaire and follow-ups patiently and carefully. The present study was supported by the Science and Technology Project of Heilongjiang province Education Department (C.W., G.F., R.X., J.W., L.W., grant no. 12531375).

\section{References}

1. Ferlay J, Shin HR, Bray F, Forman D, Mathers C and Parkin DM: Estimates of worldwide burden of cancer in 2008: GLOBOCAN 2008. Int J Cancer 127: 2893-2917, 2010.

2. Du LB, Mao WM, Chen WQ, Zhang SW, Yu CD, Zheng RS, Xia QM and Wang XH: Incidence and mortality of larynx cancer in China during 2003-2007. Zhonghua Liu Xing Bing Xue Za Zhi 33: 395-398, 2012 (In Chinese)

3. Tomatis L: Between the body and the mind: The involvement of psychological factors in the development of multifactorial diseases. Eur J Cancer 37 (Suppl 8): S148-S152, 2001.
4. Conway DI, McKinney PA, McMahon AD, Ahrens W, Schmeisser N, Benhamou S, Bouchardy C, Macfarlane GJ, Macfarlane TV, Lagiou P, et al: Socioeconomic factors associated with risk of upper aerodigestive tract cancer in Europe. Eur J Cancer 46: 588-598, 2010.

5. Santi I, Kroll LE, Dietz A, Becher H and Ramroth H: To what degree is the association between educational inequality and laryngeal cancer explained by smoking, alcohol consumption and occupational exposure? Scand J Work Environ Health 40: 315-322, 2014

6. Reiche EM, Nunes SO and Morimoto HK: Stress, depression, the immune system and cancer. Lancet Oncol 5: 617-625, 2004.

7. Lillberg K, Verkasalo PK, Kaprio J, Teppo L, Helenius H and Koskenvuo M: Stressful life events and risk of breast cancer in 10,808 women: A cohort study. Am J Epidemiol 157: 415-423, 2003.

8. Fox CM, Harper AP, Hyner GC and Lyle RM: Loneliness, emotional repression, marital quality, and major life events in women who develop breast cancer. J Community Health 19: 467-482, 1994

9. Ollonen P, Lehtonen J and Eskelinen M: Stressful and adverse life experiences in patients with breast symptoms: A prospective case-control study in Kuopio, Finland. Anticancer Res 25: 531-536, 2005.

10. Hassan S, Karpova Y, Baiz D, Yancey D, Pullikuth A, Flores A, Register T, Cline JM, D'Agostino R Jr, Danial N, et al: Behavioral stress accelerates prostate cancer development in mice. J Clin Invest 123: 874-886, 2013.

11. Azizi $\mathrm{H}$ and Esmaeili ED: Stressful life events and risk of colorectal cancer: A case-control study of Iran. Asian Pac J Cancer Prev 16: 2403-2407, 2015.

12. Yang D and Zhang Y: LES (Life Events Scale). Mental Health Rating Scale Manual. Health Mag 102-106, 1999 (In Chinese).

13. Holmes TH and Rahe RH: The social readjustment rating scale. J Psychosom Res 11: 213-218, 1967.

14. Eysenck HJ and Eysenck SB: Eysenck Personality Questionnaire (Junior and Adult). Br J Med Psychol 50: 203-205, 1977.

15. Garssen B: Psychological factors and cancer development: Evidence after 30 years of research. Clin Psychol Rev 24: 315-338, 2004.

16. Desai MM, Bruce ML and Kasl SV: The effects of major depression and phobia on stage at diagnosis of breast cancer. Int J Psychiatry Med 29: 29-45, 1999.

17. Levav I, Kohn R, Iscovich J, Abramson JH, Tsai WY and Vigdorovich D: Cancer incidence and survival following bereavement. Am J Public Health 90: 1601-1607, 2000.

18. Trikas P, Vlachonikolis I, Samonis G, Askoxilakis I, Tsiftsis D, Koutsoubi K and Paritsis N: Low psychopathology scores in the prognosis of breast cancer. A preliminary report. Psychother Psychosom 71: 162-167, 2002.

19. Tschuschke V, Hertenstein B, Arnold R, Bunjes D, Denzinger R and Kaechele $\mathrm{H}$ : Associations between coping and survival time of adult leukemia patients receiving allogeneic bone marrow transplantation-Results of a prospective study. J Psychosom Res 50: 277-285, 2001

20. Weihs KL, Enright TM, Simmens SJ and Reiss D: Negative affectivity, restriction of emotions, and site of metastases predict mortality in recurrent breast cancer. J Psychosom Res 49: 59-68, 2000.

21. Butow PN, Coates AS and Dunn SM: Psychosocial predictors of survival: Metastatic breast cancer. Ann Oncol 11: 469-474, 2000

22. Goodkin K, Antoni MH and Blaney PH: Stress and hopelessness in the promotion of cervical intraepithelial neoplasia to invasive squamous cell carcinoma of the cervix. J Psychosom Res 30: 67-76, 1986.

23. Martikainen P and Valkonen T: Mortality after the death of a spouse: Rates and causes of death in a large Finnish cohort. Am J Public Health 86: 1087-1093, 1996.

24. Shaffer JW, Duszynski KR and Thomas CB: Family attitudes in youth as a possible precursor of cancer among physicians: A search for explanatory mechanisms. J Behav Med 5: 143-163, 1982.

25. Reynolds P and Kaplan GA: Social connections and risk for cancer: Prospective evidence from the Alameda County study. Behav Med 16: 101-110, 1990.

26. Levy SM, Herberman RB, Lippman M, D'Angelo T and Lee J: Immunological and psychosocial predictors of disease recurrence in patients with early-stage breast cancer. Behav Med 17: 67-75, 1991.

27. Maunsell E, Brisson J and Deschênes L: Social support and survival among women with breast cancer. Cancer 76: 631-637, 1995. 
28. Nakaya N, Hansen PE, Schapiro IR, Eplov LF, Saito-Nakaya K, Uchitomi $\mathrm{Y}$ and Johansen C: Personality traits and cancer survival: A Danish cohort study. Br J Cancer 95: 146-152, 2006.

29. Arai S, Nakaya N, Kakizaki M, Ohmori-Matsuda K, Shimazu T, Kuriyama S, Fukao A and Tsuji I: Personality and gastric cancer screening attendance: A cross-sectional analysis from the Miyagi Cohort Study. J Epidemiol 19: 34-40, 2009.

30. van den Bergh RC, Essink-Bot ML, Roobol MJ, Wolters T, Schröder FH, Bangma CH and Steyerberg EW: Anxiety and distress during active surveillance for early prostate cancer. Cancer 115: 3868-3878, 2009.

31. Yamaoka K, Shigehisa T, Ogoshi K, Haruyama K, Watanabe M, Hayashi F and Hayashi C: Health-related quality of life varies with personality types: A comparison among cancer patients, non-cancer patients and healthy individuals in a Japanese population. Qual Life Res 7: 535-544, 1998.

32. Boscolo P: Effects of occupational stress and job insecurity on the immune response. G Ital Med Lav Ergon 31: 277-280, 2009 (In Italian).
33. Palermo-Neto J, de Oliveira Massoco C and Robespierre de Souza W: Effects of physical and psychological stressors on behavior, macrophage activity, and Ehrlich tumor growth. Brain Behav Immun 17: 43-54, 2003.

34. Hofs $\varnothing$ K, Bjordal K, Diep LM and Rustøen T: The relationships between demographic and clinical characteristics and quality of life during and after radiotherapy: In women with breast cancer. Qual Life Res 23: 2769-2777, 2014.

35. Helgeson VS, Cohen S, Schulz R and Yasko J: Group support interventions for women with breast cancer: Who benefits from what? Health Psychol 19: 107-114, 2000. 